\title{
Gravitational Mass Dependence on Velocity and the Conservation of Energy in General Relativity
}

\author{
Jaroslav Hynecek ${ }^{1}$ \\ ${ }^{1}$ Isetex, Inc., 905 Pampa Drive, Allen, TX 75013, USA \\ Correspondence: Jaroslav Hynecek, Isetex, Inc., 905 Pampa Drive, Allen, TX 75013, USA. E-mail: \\ jhynecek@netscape.net
}

Received: March 6, 2018

Accepted: March 14, 2018

Online Published: March 21, 2018

doi:10.5539/apr.v10n2p26

URL: https://doi.org/10.5539/apr.v10n2p26

\begin{abstract}
This paper investigates by simple means the relativistic accelerated motion of a small test body in a simulated uniform gravitational like field and compares the predictions of energy loss, perhaps by radiation, obtained from the General Relativity Theory (GRT) and from the Metric Theory of Gravity (MTG). The study is first conducted in a flat Minkowski space-time with simulated constant gravitational like force and later in a true curved space-time with a metric, which, however, is not derived from the GRT. It is found that the gravitational mass dependence on velocity in GRT is not correct, because it predicts a negative loss of energy while the MTG predicts correctly a positive loss. The energy is conserved in a curved space-time free fall where the gravitational mass does not depend on velocity. There can be no energy radiation during the test body free fall in a uniform gravitational field.
\end{abstract}

Keywords: General Relativity Theory, Metric Theory of Gravity, gravitational mass dependence on velocity, conservation of energy

\section{Introduction}

The theories describing the accelerated motion are well understood in both; the GRT and the MTG. In the GRT the inertial mass and the gravitational mass are assumed identical with identical dependencies on velocity. In the MTG, on the other hand, the gravitational mass depends on velocity differently than the inertial mass (Hynecek, 2005, 2017). In order to use equations from the theory that is valid in a flat Minkowski space-time it will be considered first that the uniform gravitational like force can be simulated by an idealized hypothetical rocket motor that is having a constant thrust. The curved space-time, the GRT like theory, will be discussed later for a comparison. It is thus simple for both theories and the curved space-time theory to derive equations describing the simulated free fall like velocities and from that the energy loss of a small test body that moves under a uniform acceleration force.

\section{Theories}

In the theory that works in a flat space-time, but includes acceleration, the relation between the velocity $v$ and time $t$ is somewhat more complicated but can easily be derived as follows:

$$
\frac{d}{d t}\left[\frac{m_{0} v}{\sqrt{1-v^{2} / c^{2}}}\right]=g \frac{m_{0}}{\sqrt{1-v^{2} / c^{2}}}
$$

where $m_{0}$ is the rest mass, $c$ the speed of light in a vacuum, and $g$ the simulated gravitational like acceleration constant. The left hand side of Eq. 1 is the relativistic formula for the inertial force and the right hand side is the formula for the simulated gravitational force that includes the gravitational mass dependence on velocity. The formula in Eq. 1 can be rearranged and simplified resulting in the following relation for the small body acceleration:

$$
\frac{d v}{d t}=g\left(1-v^{2} / c^{2}\right)
$$

The energy loss will be calculated by comparing the potential energy that would be exerted by lifting the small test body very slowly in the uniform gravitational like field $g$ by a distance $z$ to the energy that would be gained back when the body would "fall" the same distance $z$. The test body potential energy is simply expressed as follows:

$$
E=m_{0} g \cdot z
$$


This relation will be kept as reference energy. For the actual accelerated falling like motion of a test body the incremental energy gain can be expressed in terms of the velocity $v$ and the gravitational like force $F$ as follows:

$$
d E=F \cdot v \cdot \frac{d v}{(d v / d t)}=\frac{g \cdot m_{0}}{\sqrt{1-v^{2} / c^{2}}} \frac{v \cdot d v}{g\left(1-v^{2} / c^{2}\right)}
$$

After rearrangements and integration the expression for the energy as a function of velocity becomes:

$$
E=\frac{m_{0} c^{2}}{\sqrt{1-v^{2} / c^{2}}}
$$

This result is expected and it is a nice confirmation of methodology used in Eq.1.

In the next step we will evaluate the energy difference $\Delta E$ given by Eq.3 and Eq.5. However, to accomplish this task it is necessary to express the energy of the "falling like" body in terms of the travelled distance $z$. This can be derived starting from Eq.2 as follows:

$$
\frac{d v}{\left(1-v^{2} / c^{2}\right)}=g \frac{d t}{d z} d z
$$

After multiplying both sides of Eq. 6 by velocity $v=d z / d t$, after integration, and after rearrangements the relation between the travelled distance $z$ during the accelerated motion and the velocity becomes equal to:

$$
1-\frac{v^{2}}{c^{2}}=e^{\frac{-2 g \cdot z}{c^{2}}}
$$

Using this relation in the formula for the energy difference the result becomes as follows:

$$
\Delta E=m_{0} g \cdot z+m_{0} c^{2}-m_{0} c^{2} e^{\frac{g \cdot z}{c^{2}}}=m_{0} g \cdot z+m_{0} c^{2}-m_{0} c^{2}\left(1+\frac{g \cdot z}{c^{2}}+\frac{1}{2}\left(\frac{g \cdot z}{c^{2}}\right)^{2}+\cdots\right)
$$

In this equation the rest mass equivalent energy was added to the reference energy to be consistent with the rest energy of the accelerated body. It is necessary that for the zero distance the energy difference and the velocity of the test body are both zero. By expanding the result into a power series as is shown in Eq.8 and neglecting the higher order terms the energy loss will be as follows:

$$
\Delta E \cong-\frac{m_{0} c^{2}}{8}\left(\frac{2 g \cdot z}{c^{2}}\right)^{2} \cong-\frac{m_{0} c^{2}}{8}\left(\frac{v^{2}}{c^{2}}\right)^{2}
$$

This is a very strange result. It seems that the accelerated body is gaining some additional energy from an unknown source on top of the energy that is predicted from Eq.5. This is not reasonable and it is pointing to a problem that exists in the GRT for a long time. The gravitational mass cannot depend on velocity the same way as the inertial mass. This problem will become clear from the result presented next.

The similar expression introduced in Eq. 1 is used, but with the gravitational mass depending on velocity according to the following relation (Hynecek, 2005, 2017):

$$
m_{g}=m_{0} \sqrt{1-v^{2} / c^{2}}
$$

This leads to the following formula:

$$
\frac{d}{d t}\left[\frac{m_{0} v}{\sqrt{1-v^{2} / c^{2}}}\right]=g \cdot m_{0} \sqrt{1-\frac{v^{2}}{c^{2}}}
$$

After rearrangements the formula is simplified with the result as follows:

$$
\frac{d v}{d t}=g\left(1-v^{2} / c^{2}\right)^{2}
$$

Following the same procedure as above for the GRT type of mass dependence on velocity the differential of energy will be:

$$
d E=g \cdot m_{0} \sqrt{1-\frac{v^{2}}{c^{2}}} \frac{v \cdot d v}{g\left(1-v^{2} / c^{2}\right)^{2}}
$$


This relation becomes, after integration, identical to formula in Eq.5. Both theories, the GRT and the MTG, thus give the same expression for the energy, which is expected and confirms once more that the calculating procedure is correct. For the calculation of energy loss the same procedure will also be followed as before. From Eq.12 the relation between the distance and velocity is:

$$
\frac{d v}{\left(1-v^{2} / c^{2}\right)^{2}}=g \frac{d t}{d z} d z
$$

which becomes after multiplying both sides of equation by velocity and after integration equal to:

$$
\frac{1}{\left(1-v^{2} / c^{2}\right)}=1+\frac{2 g \cdot z}{c^{2}}
$$

The energy loss difference is then calculated also as before. By adding the rest mass energy to the reference results in the following:

$$
\Delta E=m_{0} g \cdot z+m_{0} c^{2}-m_{0} c^{2} \sqrt{1+\frac{2 g \cdot z}{c^{2}}}=m_{0} g \cdot z+m_{0} c^{2}-m_{0} c^{2}\left(1+\frac{g \cdot z}{c^{2}}-\frac{1}{2}\left(\frac{g \cdot z}{c^{2}}\right)^{2}+\cdots\right)
$$

Again, by using the expansion of this result into a power series and neglecting the higher order terms the energy loss becomes equal to:

$$
\Delta E \cong \frac{m_{0} c^{2}}{8}\left(\frac{2 g \cdot z}{c^{2}}\right)^{2} \cong \frac{m_{0} c^{2}}{8}\left(\frac{v^{2}}{c^{2}}\right)^{2}
$$

This is a similar result as in Eq.9 except that the energy loss is now positive as it should be. This confirms the correctness of the gravitational mass dependence on velocity in the MTG and, therefore, soundly disproves the validity of the GRT mass dependence on velocity. The positive energy loss is justifiable by a gravitational wave radiation and the force of its radiation reaction because the body is accelerated. However, it is difficult to justify the negative energy loss as is derived in the GRT. Where does the energy come from?

From the above derivations it is clear that the root cause of this problem is the incorrect dependency of gravitational mass on velocity in GRT. The absolute identity of inertial and gravitation masses for all the velocities as postulated in an ad hoc fashion in that theory is not sustainable. Fortunately the MTG eliminates this problem and it seems that this is the correct theory of gravity.

For a better understanding of the magnitude of the radiated energy the graph of the energy loss as a function of the travelled distance during the accelerated motion for a mass of $10,000 \mathrm{~kg}$ is shown in Figure 1.

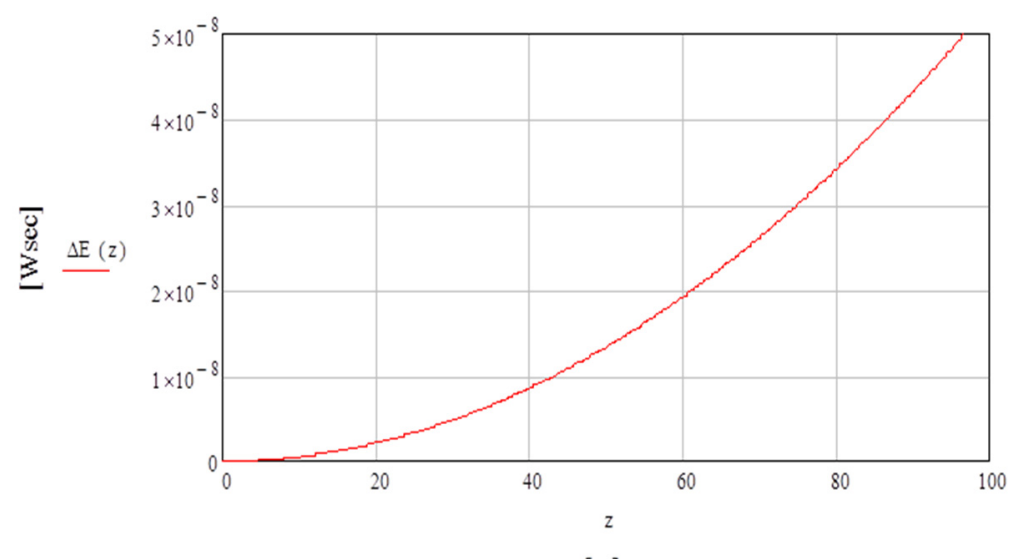

[m]

Figure 1. The dependency of energy loss due to radiation for a $10,000 \mathrm{~kg}$ test body that is being accelerated in a simulated free fall by a constant force equal to the Earth's gravity on the travelled distance $z$

However, a valid objection to the above analysis can be made that the actual gravitational field distorts the space-time and this needs to be included in the analysis. The metric for such a true uniform gravitational field space-time, however, is not the GRT based metric but has been derived elsewhere (Hynecek, 2009) as follows: 


$$
d s^{2}=\frac{(c d t)^{2}}{\left(1+g \cdot z / c^{2}\right)^{2}}-\frac{d z^{2}}{\left(1+g \cdot z / c^{2}\right)^{2}}
$$

For this case it was shown using the Lagrange formalism to analyze the free fall motion that the gravitational mass must stay constant and, therefore, does not depend on velocity at all. The force balance equation is thus as follows:

$$
\frac{d}{d t}\left[\frac{m_{0} v}{\sqrt{1-v^{2} / c^{2}}}\right]=g \cdot m_{0}
$$

This results in the following equation for the acceleration:

$$
\frac{d v}{d t}=g\left(1-v^{2} / c^{2}\right)^{3 / 2}
$$

Similarly as in Eq.14 this equation can be rearranged as follows:

$$
\frac{d v}{\left(1-v^{2} / c^{2}\right)^{3 / 2}}=g \frac{d t}{d z} d z
$$

which again by multiplication of both sides by velocity and after integration changes into the following:

$$
\frac{1}{\sqrt{1-v^{2} / c^{2}}}=1+\frac{g \cdot z}{c^{2}}
$$

By multiplying both sides of this equation by $m_{0} c^{2}$ the result is:

$$
\frac{m_{0} c^{2}}{\sqrt{1-v^{2} / c^{2}}}=m_{0} c^{2}+m_{0} g \cdot z
$$

This equation clearly states that the energy is conserved and no loss occurs during the actual fall. The free falling body in a uniform gravitational field, therefore, does not radiate any energy. The accelerated motion of a test body in a Minkowski flat space-time, on the other hand, radiates energy according to MTG, but incorrectly according to the GRT. The reason is that the gravitational mass is not equal to the inertial mass as is assumed in the GRT.

\section{Conclusions}

The paper derived simple expressions for the energy loss during the test body accelerated motion in a simulated uniform gravitational like field. It was shown that the loss derived according to the GRT mass dependence on velocity is negative. This is unacceptable and this fact disproves the validity of the GRT gravitational mass dependency on velocity.

When the different dependency of gravitational mass on velocity is used, as is derived in the MTG, the expected positive energy loss is calculated.

For the case when no energy loss is found for a mass falling in a true and not simulated uniform gravitational field the gravitational mass must stay independent of velocity in order that the law of energy conservation is satisfied.

The presented results have fatal consequences for the GRT, because unquestionably prove its incorrectness. These findings thus have a significant impact on all the theories based on the GRT such as the Big Bang and similar ridiculous models of the Universe.

\section{References}

Hynecek, J. (2005). Remarks on the Equivalence of Inertial and Gravitational Masses and on the Accuracy of Einstein's Theory of Gravity. Physics Essays, 18(2).

Hynecek, J. (2009). New space-time metrics for symmetric spaces. Physics Essays, 22(2).

Hynecek, J. (2017). Review of Internal Inconsistencies in GRT and Comparisons to MTG. The general science journal. Retrieved from http://gsjournal.net/Science-Journals/Research\%20Papers/View/7070

\section{Copyrights}

Copyright for this article is retained by the author(s), with first publication rights granted to the journal.

This is an open-access article distributed under the terms and conditions of the Creative Commons Attribution license (http://creativecommons.org/licenses/by/4.0/). 